\title{
Graduated characterization method using a multi-well microplate for reducing reactivity of nanoscale zero valent iron materials
}

Hwang, Yuhoon; Salatas, Apostolos; Mines, Paul D.; Jakobsen, Mogens Havsteen; Andersen, Henrik Rasmus

Published in:

Applied Catalysis B: Environmental

Link to article, DOI:

10.1016/j.apcatb.2015.07.041

Publication date:

2015

Document Version

Peer reviewed version

Link back to DTU Orbit

Citation (APA):

Hwang, Y., Salatas, A., Mines, P. D., Jakobsen, M. H., \& Andersen, H. R. (2015). Graduated characterization method using a multi-well microplate for reducing reactivity of nanoscale zero valent iron materials. Applied Catalysis B: Environmental, 181, 314-320. https://doi.org/10.1016/j.apcatb.2015.07.041

\section{General rights}

Copyright and moral rights for the publications made accessible in the public portal are retained by the authors and/or other copyright owners and it is a condition of accessing publications that users recognise and abide by the legal requirements associated with these rights.

- Users may download and print one copy of any publication from the public portal for the purpose of private study or research.

- You may not further distribute the material or use it for any profit-making activity or commercial gain

- You may freely distribute the URL identifying the publication in the public portal 


\title{
Graduated characterization method using a multi-well microplate for reducing reactivity of nanoscale zero valent iron materials
}

\author{
Yuhoon Hwang $^{1 *}$, Apostolos Salatas ${ }^{1,2}$, Paul D. Mines ${ }^{1}$, Mogens H. Jakobsen ${ }^{3}$, Henrik R. Andersen ${ }^{1}$ \\ ${ }^{1}$ Department of Environmental Engineering, Technical University of Denmark, Miljøvej, B113, DK-2800 Kgs. Lyngby, Denmark \\ ${ }^{2}$ Department of Environment, University of the Aegean, Xenia Building, University Hill, 81100 Mytilene, Greece \\ ${ }^{3}$ Department of Micro- and Nanotechnology, Technical University of Denmark, Ørsteds Plads, B 345E, DK-2800 Kgs. Lyngby, \\ Denmark
}

\section{Highlights}

Color assays using five substrates characterized a graduated reactivity order of nZVI.

- The assays quantified reduction products to avoid interfering reactions.

Three reaction products were quantified using both the same wavelength and reagents.

Assay was adapted to a 96-well microplate, thus minimizing sample and reagent use.

Step 1

Batch test with a range of substrate

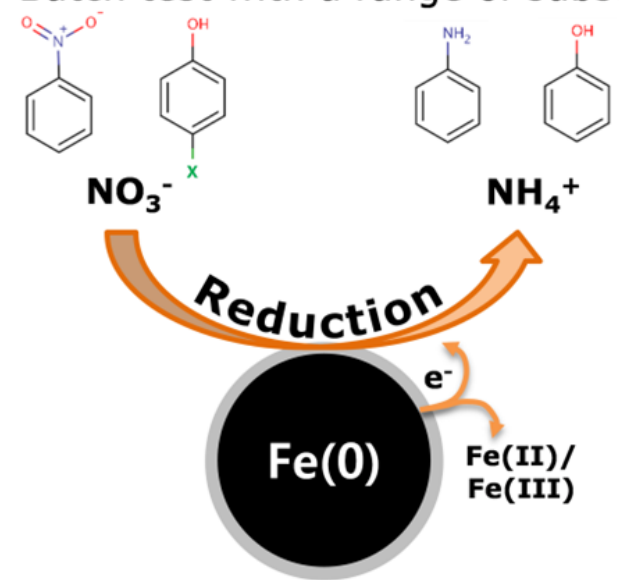

Step 2

Color assay for reaction product

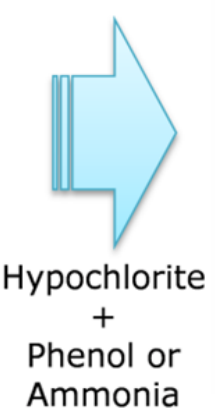

Ammonia

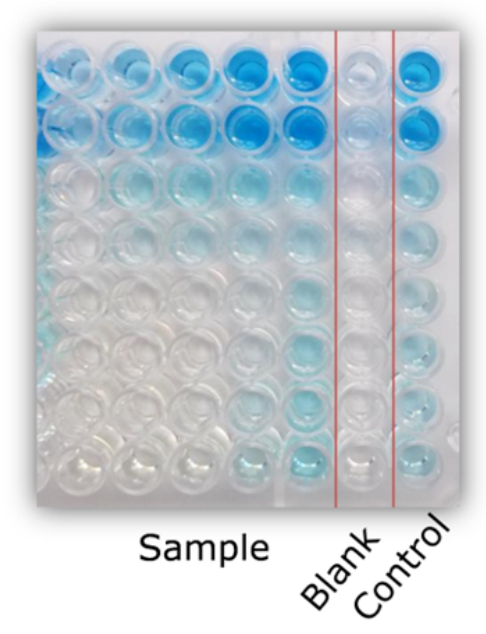

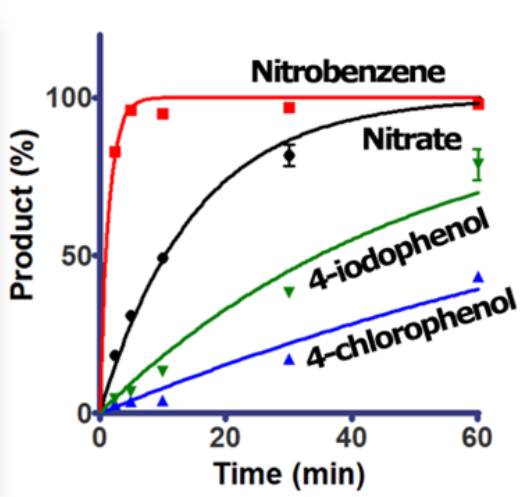

Time (min)

\begin{abstract}
Even though nanoscale zero valent iron (nZVI) has been intensively studied for the treatment of a plethora of pollutants through reductive reaction, quantification of nZVI reactivity has not yet been standardized. Here, we adapted colorimetric assays for determining reductive activity of nZVI and its composites with other metals. The assay quantifies reduction products to avoid interfering reactions, such as sorption and volatilization. Three different reaction products, ammonium, phenol, and aniline, generated as the result of reduction of nitrate, p-halophenols, and nitrobenzene, respectively, could be quantified using the same reagent for all reactions. The colorimetric assays were further adapted to the 96-well microplate format, thus minimizing sample and reagent use, as well as lowering color development time to $2 \mathrm{~h}$. The substrates showed graduated reactivity and thus reduction potency and kinetics of different materials and reaction mechanism was distinguished. The applicability was successfully proven by determining the reactivity of a commercial nZVI sample, and investigating the effect of nickel content on dehalogenation. Therefore, the suggested reactivity test with different compounds, combined with the use of a multi-well microplate based color assay, promises to be a useful and simple tool in various nZVI related research topics.
\end{abstract}

Keywords - Nanoscale zero valent iron; Indophenol reaction; Dehalogenation reactivity; Colorimetric assay; Multi-well microplate 


\section{INTRODUCTION}

Nanoscale zero valent iron (nZVI) has been intensively studied for environmental remediation, due to its strong reductive power and reactivity, which is derived from its small size and high specific surface area [1]. Its standard redox potential $\left(\mathrm{E}^{0}=-0.44 \mathrm{~V}\right)$ makes $\mathrm{nZVI}$ an effective material when reacting with reducible contaminants. The removal mechanism by nZVI involves the direct transfer of electrons to the contaminants, transforming them into non-toxic, less toxic, or more biodegradable species [2,3]. A wide variety of pollutants, including halogenated organics, nitro aromatic compounds, heavy metals, and oxy-anions, have been successfully remediated, by utilizing the reduction reaction mechanism [4].

Although a number of different nZVI materials have been prepared and compared, across many intensive studies, different properties of prepared nZVIs make it hard to compare and standardize. Depending on the aim of research and laboratory environment, the synthesis conditions of nZVI can be significantly varied; and, with it brings about considerably different nZVI properties. The effect of synthesis conditions, such as reduction time and precursor concentration, on nZVI characteristics and reactivity has been previously investigated [5]. It has also been determined that nZVI properties can be greatly influenced by solvent conditions during synthesis [6,7]. Moreover, surface modification of nZVI has been intentionally performed, in order to enhance nZVI properties desirable for environmental remediation. In order to prevent aggregation for in situ subsurface remediation, particle stabilization or nZVI immobilization on various support materials has been achieved in many studies [8,9]. Recently, remarkable enhancement of stability and reactivity was reported by applying an organo-functionalized clay, i.e. aminoclay $[10,11]$. Additionally, bimetallic particles with iron as the primary metal and the deposition of a thin layer of noble or transition metals, such as palladium (Pd), copper $(\mathrm{Cu})$, nickel $(\mathrm{Ni})$ or platinum $(\mathrm{Pt})$ on the iron surface has been frequently applied to enhance the reactivity of nZVI, especially for reductive dehalogenation [12-14].

However, the evaluation of nZVI reactivity towards dehalogenation has not yet been standardized. The use of advanced chromatographic techniques has been the predominant method to determine the treatment target and subsequent degradation products $[15,16]$. These techniques are powerful tools to analyze the compounds, both qualitatively and quantitatively. At the same time, it generally requires lengthy analysis procedures and complicated set-ups. In this light, a simple color assay for use in laboratories, requiring only a basic spectrophotometer for determining color intensity would be a very useful tool.

In our previous study, the modified indophenol method for detecting phenol, which is the result from dehalogenation of 4-chlorophenol, was suggested to determine nZVI reactivity [17]. The aforementioned method was successfully applied to determine dehalogenation reactivity of lab synthesized monometallic and bimetallic nZVI. Throughout the previous study, analysis was focused on determination of the reduction product, rather than the treatment target. This gave a more accurate observation of the reducing activity of nZVI, due to the fact that other reactions, e.g. sorption, oxidation, or co-precipitation cannot interfere. In this study, an extended range of substrates, i.e. nitrate, nitrobenzene, and other halophenols (4-bromophenol and 4-iodophenol), was applied to give better understanding of nZVI reactivity. It was hypothesized that the reduction kinetics and capacity of these substrates would be different, depending on the involved redox reaction and chemical bond. Therefore, the graduated reducing reactivity towards different reducible compounds can be used to determine reduction kinetics and capacity.

Herein, the colorimetric assay to determine nZVI reactivity was further developed and optimized through a multi-well microplate based analysis. Firstly, the previously developed phenol detection method was further miniaturized and optimized for quantification in a 96-well microplate. The expanded range of target compounds, e.g. nitrate, nitrobenzene, and parapositioned halogenated phenols, which can be analyzed by the same reagent to create color, was applied, and subsequently optimized in the same manner. The range of target compounds provided a graduated characterization of reducing activity, with respect to our previously published method using 4-chlorophenol. Lastly, the applicability of the suggested method for investigation on the effect of different factors during the reaction and production of nZVI was demonstrated. Based on the overall results, a simple and effective assay to determine nZVI reactivity is suggested.

\section{METHODS}

\subsection{Color assay for analyzing reaction products}

In this study, the same regime of reagents was applied to ammonium, phenol, and aniline to create a colored product. The use of similar reagents gives simplicity and versatility on analyzing several reaction products at the same time. The following sections describe in-detail procedures of the color assays.

\subsubsection{Ammonium analysis as a product of nitrate reduction}

The indophenol reaction is the name given to the reaction of monochloramine and a phenol, which results in the formation of a blue indophenol dye, which can then be determined spectrophotometrically $[18,19]$. This reaction is highly specific for ammonium, and in the presence of a suitable catalyst, extremely high sensitivity is attainable. Ammonium analysis was conducted according to standard methods, with a slight modification (4500- $\mathrm{NH}_{3}$-D) [20] Basically, this consisted of ammonium measurement, using ammonium as a limiting reagent, with excess amounts of phenol and 
hypochlorite being applied to ensure all ammonium forms indophenol, when catalyzed by sodium nitroprusside. Reagent A was prepared by diluting $1 \mathrm{~mL}$ of $10 \%$ hypochlorite solution and $66 \mathrm{~mL}$ of $0.5 \mathrm{M} \mathrm{NaOH}$ solution to $100 \mathrm{~mL}$ with deionized water. Reagent B was prepared as $26 \mathrm{~g} / \mathrm{L}$ of phenol and $0.3 \mathrm{~g} / \mathrm{L}$ of sodium nitroprusside. Samples of $2.3 \mathrm{~mL}$ are transferred to a cuvette and $0.1 \mathrm{~mL}$ of each reagent is added to the sample. The mixture is then allowed to react in the dark for $2 \mathrm{~h}$ and subsequently quantified with a UV-Vis spectrophotometer (Cary 50, Agilent Technologies Inc., USA) at $630 \mathrm{~nm}$.

\subsubsection{Phenol analysis as a product of halogenated phenol reduction}

In our previous study, phenol detection by the indophenol reaction was successfully developed by simple substitution of phenol as the limiting substrate in the entire reaction [17]. In this color assay, phenol acted as the limiting reagent, and excess amounts of ammonium and hypochlorite were applied, in order to have the color development dependent on the concentration of phenol in samples. For the color assay, Reagent A was modified to have a lower concentration of hypochlorite, which was optimized as described in section 2.2.2. Reagent A was prepared by diluting 0.2 $\mathrm{mL}$ of $10 \%$ hypochlorite solution and $66 \mathrm{~mL}$ of $0.5 \mathrm{M}$ $\mathrm{NaOH}$ solution to $100 \mathrm{~mL}$ with deionized water. Reagent $\mathrm{C}$ was prepared as $100 \mathrm{mg}-\mathrm{NH}_{4}{ }^{+}-\mathrm{N} / \mathrm{L}$ of ammonium chloride and $0.3 \mathrm{~g} / \mathrm{L}$ of sodium nitroprusside in deionized water. A drop of hydrochloric acid was added into Reagent $\mathrm{C}$ to minimize ammonia stripping into the headspace. Equal volumes of reagents $\mathrm{A}$ and $\mathrm{C}$ were premixed to make the reagent $\mathrm{AC}$. Then, $2.3 \mathrm{~mL}$ of sample was transferred into a cuvette, and $0.2 \mathrm{~mL}$ of reagent AC was added to the sample. The mixture was allowed to react in the dark for $2 \mathrm{~h}$ and subsequently quantified with a spectrometer at $680 \mathrm{~nm}$.

\subsubsection{Aniline analysis as a product of nitrobenzene reduction}

Reagents $\mathrm{A}$ and $\mathrm{C}$ were used for aniline analysis, as described in section 2.1.2. In short, $0.1 \mathrm{~mL}$ of reagent $\mathrm{A}$ was added to $2.3 \mathrm{~mL}$ of the sample, and subsequently 0.1 $\mathrm{mL}$ of reagent $\mathrm{C}$ was added after $15 \mathrm{~min}$ of reaction. The mixture allowed to react in the dark for $2 \mathrm{~h}$ and then quantified with a spectrometer at $680 \mathrm{~nm}$.

\subsection{Miniaturization and optimization for quantification in 96-well microplate.}

\subsubsection{Use of multiwell plate and plate reader for} simple analysis

The multi-well microplate was applied in order to simplify the analysis procedure. Using a multi-well microplate offers the advantage of reading multiple samples with an automated plate reader and having a small sample volume for quantification. In this study, the samples were prepared in a 96-well plate (Nunc polypropylene flat bottom microwell plate, Thermo Fisher Scientific, Inc. USA) and were measured over the range of 400-800 nm, using a Synergy MX microplate reader (BioTek Instruments, Inc., USA). The 96-well plates can hold a maximum volume of approximately $400 \mu \mathrm{L}$. In our assays, the volume of the sample was 230 $\mu \mathrm{L}$ and $10 \mu \mathrm{L}$ for each of the reagents, which is an order of magnitude less volume than when using a normal cuvette. The remaining space in the well was assessed as sufficient for mixing of the mixture.

\subsubsection{Optimization of reaction conditions}

In order to evaluate the optimal conditions for the reagents, we tested the color assay at different concentration levels of active chlorine for reagent $\mathrm{A}$, $0.01-0.4 \%$ active chlorine, through adding $0.1-4 \mathrm{~mL}$ of $10 \%$ hypochlorite solution in $100 \mathrm{~mL}$ reagent $\mathrm{A}$ and ammonium for reagent $\mathrm{C}\left(50-5000 \mathrm{mg}-\mathrm{NH}_{4}{ }^{+}-\mathrm{N} / \mathrm{L}\right)$. The reaction time was also further optimized by obtaining the calibration curve at different time intervals, up to $24 \mathrm{~h}$. Since ammonium analysis has already been standardized and optimized as a standard method, further optimization was not considered.

\subsubsection{Limit of detection and quantification of analytical methods}

The calibration curve was acquired by linear regression analysis. The limit of detection (LOD) was calculated as 3 times the standard deviation of the lowest concentration and the limit of quantification (LOQ) as 10 times of the standard deviation of the lowest concentration. Sample absorbance was corrected for reagent blanks and concentrations calculated using a standard curve slope. The tested concentration ranges were (i) for phenol 0-50 $\mu \mathrm{M}$, (ii) for aniline $0-75 \mu \mathrm{M}$ and (iii) for ammonium 0-2 mg- $\mathrm{NH}_{4}{ }^{+}-\mathrm{N} / \mathrm{L}(0-143 \mu \mathrm{M})$.

\subsection{Preparation of $n Z V I$ and bimetallic nZVI}

\subsubsection{Commercial nZVI particles}

Commercially available air-stable nZVI powder (NANOFER STAR) was obtained from Nanoiron s.r.o (Czech Republic). The powder was delivered in an airtight vial from the manufacturer. Once it was opened, it was stored in an anaerobic chamber $\left(\mathrm{N}_{2}: \mathrm{H}_{2}=95: 5\right.$, Coy laboratory products, USA) to minimize risk of further oxidation.

\subsubsection{Preparation of lab synthesized nZVI and} bimetallic nZVI

In this study, the mild chemical reduction of metal salts in the solution phase was used to prepare nZVI, as stated in previous research [10]:

$$
2 \mathrm{Fe}^{2+}+\mathrm{BH}_{4}^{-}+3 \mathrm{H}_{2} \mathrm{O} \rightarrow 2 \mathrm{Fe}^{0}+\mathrm{H}_{2} \mathrm{BO}_{3}{ }^{-}+4 \mathrm{H}^{+}+2 \mathrm{H}_{2}
$$

The synthesis of nZVI was conducted in a $1000-\mathrm{mL}$ 
three-open-neck round bottom flask reactor. The central neck was housed with a tunable mechanical stirrer at 402000 rpm (RZR2021, Heidolph, Germany). The stirring speed was adjusted to $250 \mathrm{rpm}$ for nZVI synthesis, and to $100 \mathrm{rpm}$ for aging. Iron precursor solution was prepared with $600 \mathrm{~mL}$ of $0.24 \mathrm{M} \mathrm{FeSO}_{4} \cdot 7 \mathrm{H}_{2} \mathrm{O}$ in deionized water. The reduction of iron ions was instigated by introducing $200 \mathrm{~mL}$ of $1.8 \mathrm{M} \mathrm{NaBH} 4$ solution via peristaltic pump with a constant delivery rate of $10 \mathrm{~mL} / \mathrm{min}$. The black precipitate was further aged for 30 min after injection of $\mathrm{NaBH}_{4}$ solution. Collection and washing of nZVI was performed via centrifugation (2500 rpm, 5 min, Heraeus Multifuge X1, Thermo Fisher Scientific Inc., USA), and the collected nZVI particles were then washed once with deionized water and twice with absolute ethanol. The washed nZVI particles were subsequently dried in a vacuum oven at $60^{\circ} \mathrm{C}$ for $4 \mathrm{~h}$. The dried nZVI was stored in air tight vials inside of the anaerobic chamber. The physical characteristics of was not considered. prepared nZVI are considered to be identical with uncoated nZVI prepared in previous research [10], which used the same synthesis protocol with this study.

Bimetallic particles were prepared via chemical reduction, similar as with nZVI preparation [21]. The secondary metal solution was introduced to nZVI particles, and then spontaneously bimetallic particles were produced. The bimetallic nZVIs were prepared by mixing the secondary metal solution $\left(\mathrm{NiSO}_{4}\right)$ with nZVI particles. The nickel content was controlled as $0.01-5 \%$ by changing volume of nickel stock solution into reaction vials. After 10 min of redox reaction between the second metal and nZVI, the resulting $\mathrm{Ni} / \mathrm{Fe}$ bimetallic particles were collected and washed twice with deionized water. Bimetallic particles were prepared and used immediately in the reactivity test; therefore, further

drying

\subsection{Reduction of compounds by nZVIs}

Batch tests for nZVI reactivity were conducted in $50-\mathrm{mL}$ glass serum vials. The desired amount of nZVI was weighed in reaction vials inside of the anaerobic chamber. Then, reaction vials were filled with $39 \mathrm{~mL}$ of deionized water and securely capped with a PTFE/silicon septum and aluminum crimp seal. Each stock solution of $1 \mathrm{~mL}$ was injected through the septum to initiate the reaction. The nominal concentrations of nitrobenzene, nitrate, para-halogenated phenols and nZVI were 500 $\mu \mathrm{M}, \quad 25 \mathrm{mg}-\mathrm{NO}_{3}^{-}-\mathrm{N} / \mathrm{L}, \quad 500 \mu \mathrm{M}$, and $2 \mathrm{~g}-\mathrm{Fe} / \mathrm{L}$, respectively. Vials were mixed on an orbital shaker throughout the entire reaction. Samples of $4 \mathrm{~mL}$ were taken periodically and filtered with $0.45 \mu \mathrm{m}$ syringe filters. The concentration of reaction products in the sample were measured, as described in Section 2.2. Reaction kinetics were further interpreted by pseudo first-order kinetics (Eq. 2).

$$
C_{t}=C_{0} \cdot e^{-k t}
$$

Where $C_{t}$ is the concentration at the reaction time, $C_{0}$ is the initial concentration, and $\mathrm{k}$ is the reaction rate constant $\left(\mathrm{h}^{-1}\right)$.

\section{RESULTS AND DISCUSSION}

3.1 Modified indophenol reaction based color assay for analyzing reaction products in multi-well microplate

\subsubsection{Ammonium analysis}

Further optimization on reagent concentration and time for the ammonium analysis was not performed, being that it is already a standardized method. Sample and reagent volumes were decreased an order of magnitude, to fit in the wells of the multi-well microplate. The standard curve, LOD, and LOQ were obtained using the multi-well plate and plate reader, as presented in Figure $\mathrm{S} 1$ and Table 1. The values of the LOD and LOQ in $2 \mathrm{~h}$ were 2.9 and $9.6 \mu \mathrm{M}$, respectively.

\subsubsection{Phenol analysis}

Effect of the reagent concentration on phenol detection was investigated, as presented in Figure 1 (a). The maximum absorbance was obtained when using $0.02 \%$ active chlorine in reagent $\mathrm{A}$ and $100 \mathrm{mg}-\mathrm{NH}_{4}{ }^{+}-\mathrm{N} / \mathrm{L}$ in Reagent $\mathrm{C}$. It gave a nominal concentration in the mixture of sample and reagent as $9.3 \mathrm{mg} / \mathrm{L}$ of active chlorine and $4 \mathrm{mg}-\mathrm{NH}_{4}{ }^{+}-\mathrm{N} / \mathrm{L}$. This clearly demonstrated that the absorbance decreases with increasing amount of hypochlorite and increasing amount of ammonium. The excess hypochlorite will react with phenol causing the decrease of the absorbance. Moreover, more ammonium is required to get a maximum absorbance with increased hypochlorite concentration. A linear relationship could be obtained between the amount of hypochlorite and the amount of ammonium; therefore, it could be concluded that color development is closely related to the stoichiometry of the reaction. 
(a)

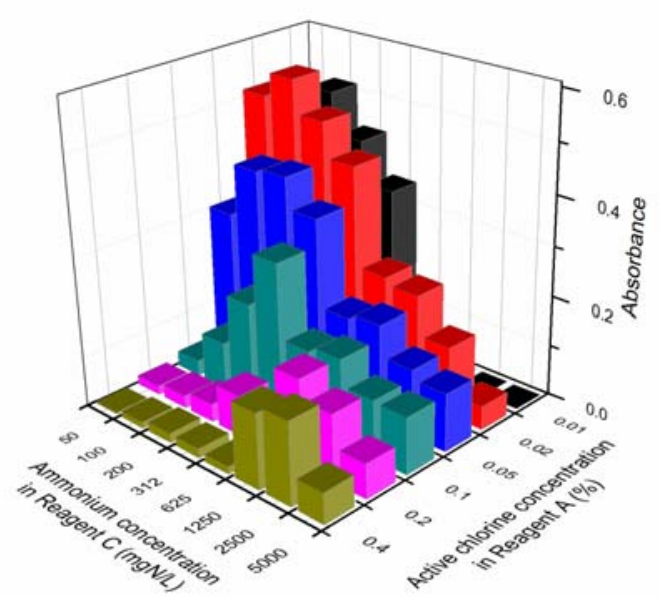

(b)

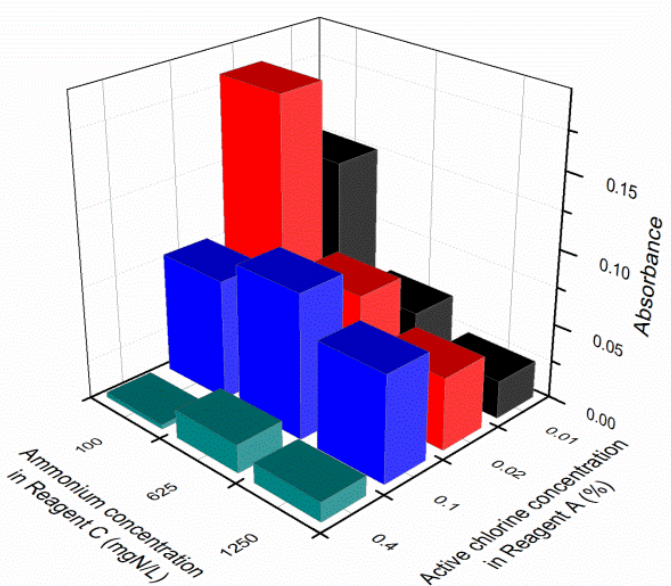

Figure 1 Optimization of reagent concentration on color development for (a) phenol, and (b) aniline. Note that $50 \mu \mathrm{M}$ of each product was applied and absorbance was measured after $2 \mathrm{~h}$ of reaction.

Effect of reaction time was also investigated, as detailed in Figure $\mathrm{S} 1$ and Table $\mathrm{S} 1$. The slope is steadily increasing during the $2 \mathrm{~h}$ of reaction, and it was stable up to $4 \mathrm{~h}$ of reaction time. The absorbance then decreased during the remainder of the $24 \mathrm{~h}$ reaction time, which indicated that the color product is not stable after $4 \mathrm{~h}$. In summary, the optimal reaction time can be determined as $2 \mathrm{~h}$, since it demonstrated comparably high absorbance and linearity, as well as a shortened reaction time within the stable time range of 2-4 h. The values of the LOD and LOQ in $2 \mathrm{~h}$ were 0.3 and $1.0 \mu \mathrm{M}$, respectively.

\subsubsection{Aniline analysis}

The suggested color assay was tested for detection of both aniline and nitrobenzene in a concentration of 50 $\mu \mathrm{M}$. The adsorption spectra of the color product by aniline and nitrobenzene are detailed in Figure S2. The reaction when applyinganiline produced a color product with a clear peak at a wavelength of $680 \mathrm{~nm}$, regardless of the presence of nitrobenzene. Thus, it was obvious that the selectivity of the color assay adequately distinguished aniline.

Figure 1 (b) outlines how the optimal reagent concentrations for aniline detection are the same as with the phenol assays, as described in section 3.1.2. The sensitivity of color development is lower than with the detection of phenol, therefore, the LOD and LOQ are slightly higher, 0.5 and $1.7 \mu \mathrm{M}$ for the LOD and LOQ, respectively. Figure S3 and Table S2 present a standard curve optained, with respect to reaction time. The slope is almost stable after a hour of reaction time, which is faster than the reaction with phenol. Since the standard curve slope and its accuracy is nearly stable after a hour of reaction, we took $2 \mathrm{~h}$ to be the standard condition, which has the advantage that phenol and aniline can be measured concurrently.

Table 1 Summary of analytical methods using a multi-well microplate for determination of reaction products.

\begin{tabular}{|c|c|c|c|}
\hline & Ammonium & Phenol & Aniline \\
\hline Sample volume $(\mu \mathrm{L})$ & 230 & 230 & 230 \\
\hline Reagents & $A+B$ & $\mathrm{AC}$ & $\mathrm{A}+\mathrm{C}(15 \mathrm{~min}$ interval $)$ \\
\hline $\begin{array}{l}\text { Concentration range } \\
\text { for analysis }(\mu \mathrm{M})\end{array}$ & $0-143\left(0-2 \mathrm{mg}-\mathrm{NH}_{4}^{+}-\mathrm{N} / \mathrm{L}\right)$ & $0-50$ & $0-75$ \\
\hline LOD $(\mu \mathrm{M})$ & 2.9 & 0.3 & 0.5 \\
\hline $\operatorname{LOQ}(\mu \mathrm{M})$ & 9.6 & 1.0 & 1.7 \\
\hline Reaction time (h) & 2 & 2 & 2 \\
\hline
\end{tabular}

3.2 Graduated characterization of reducing activity by applying a range of reducible compounds

A range of reducible compounds was tested to characterize the graduated reducing activity. In this study, nitrate, nitrobenzene, and para-halogenated phenols (i.e. 4-chlorophenol, 4-bromophenol and 4-iodophenol) were applied. Both lab-synthesized monometallic nZVI and bimetallic nZVI (Ni/Fe $5 \% \mathrm{w} / \mathrm{w})$ were applied. The iron concentration was fixed as $2 \mathrm{~g}-\mathrm{Fe} / \mathrm{L}$. 
(a)

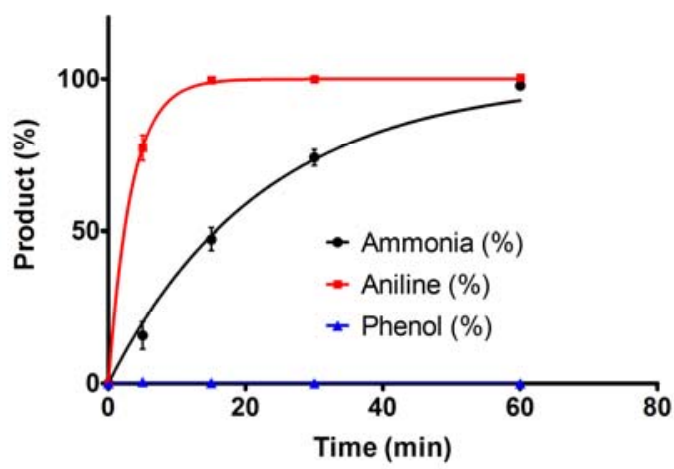

(b)

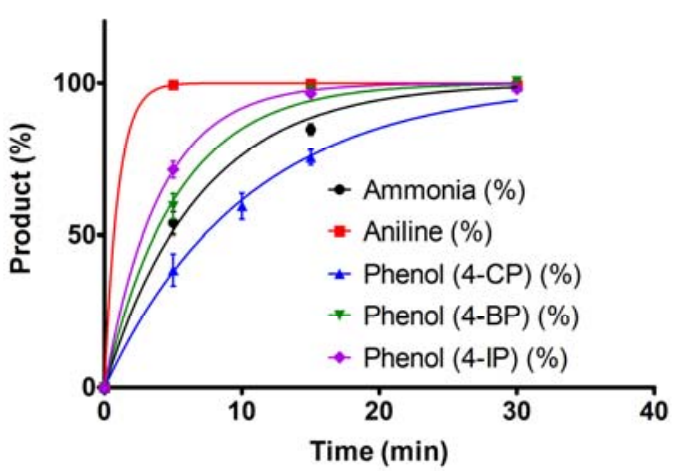

Figure 2 Generation of reaction products by reaction with (a) monometallic nZVI, and (b) bimetallic $\mathrm{Ni} / \mathrm{Fe}(5 \%)$. Note that the iron concentration in all cases was $2 \mathrm{~g} / \mathrm{L}$, and curves were fitted by pseudo first-order kinetics. Legends indicate the treatment target and its products.

Table 2 Kinetic constants and half-life time derived via curve fitting by pseudo first-order kinetics (lab synthesized nZVIs).

\begin{tabular}{c|cccc}
\hline nZVI & Compounds & Kinetic constant $\left(\boldsymbol{h}^{-\mathbf{1}}\right)$ & $\boldsymbol{t}_{\mathbf{1 / 2}}(\mathbf{m i n})$ & $\boldsymbol{R}^{\mathbf{2}}$ \\
\hline \hline \multirow{2}{*}{ Monometallic nZVI } & Nitrate & 2.65 & 15.7 & 1.00 \\
& Nitrobenzene & 17.9 & 2.32 & 1.00 \\
\hline \multirow{3}{*}{ Bimetallic nZVI } & Nitrate & 10.3 & 4.05 & 0.98 \\
(Ni/Fe 5\%) & Nitrobenzene & 93.4 & 0.445 & 0.99 \\
& 4-chlorophenol & 5.72 & 7.27 & 0.99 \\
& 4-bromophenol & 10.1 & 4.11 & 0.99 \\
\hline
\end{tabular}

The graduated reducing activity of nZVI towards different compounds is presented in Figure 2 and Table 2. Reduction kinetics were well described by a pseudo firstorder reaction. The reaction rate constant and half-life time was calculated, as is listed in Table 2. Figure 2 (a) presents the reactivity of monometallic nZVI towards different reducible compounds. As a result of nitrate and nitrobenzene reduction by nZVI, ammonium and aniline could be produced, as described in the following equations (Eq. 3-4).

$$
\begin{gathered}
4 \mathrm{Fe}^{0}+\mathrm{NO}_{3}^{-}+10 \mathrm{H}^{+} \rightarrow 4 \mathrm{Fe}^{2+}+\mathrm{NH}_{4}^{+}+3 \mathrm{H}_{2} \mathrm{O} \quad \text { (Eq. 3) } \\
\mathrm{C}_{6} \mathrm{H}_{5} \mathrm{NO}_{2}+3 \mathrm{Fe}^{0}+6 \mathrm{H}^{+} \rightarrow \mathrm{C}_{6} \mathrm{H}_{5} \mathrm{NH}_{2}+3 \mathrm{Fe}^{2+}+2 \mathrm{H}_{2} \mathrm{O}
\end{gathered}
$$

(Eq. 4)

Aniline production was the fastest reaction, and the corresponding rate constant and half-life time were calculated to be $17.9 \mathrm{~h}^{-1}$ and $2.32 \mathrm{~min}$, respectively; which is 6.75 times faster than that of nitrate. On the other hand, phenol production was not observed from any of the halogenated phenols. This result agrees with previous findings on dechlorination of chlorinated aromatic compounds by bare ZVI [22].

The overall reactions were greatly accelerated by the second metal, as presented in Figure 2 (b). The 5\% $\mathrm{Ni} /$ Febimetallic particles exhibited 3.9-5.2 times higher reaction rate constants than monometallic nZVI particles. This indicates that the addition of a second metal on the nZVI surface can promote iron corrosion, due to galvanic effects between nickel and iron. A higher standard redox potential of the second metal would develop a galvanic cell where iron corrosion is accelerated [21]. More electrons can be produced and supplied to reducible compounds, and then a faster reaction can be achieved. In addition, catalytic reactivity of the second metal, nickel was also confirmed by parapositioned halogenated phenols. Phenol was produced as a result of the catalytic reduction of para-positioned halogenated phenols, as described in the following equations (Eq. 5-7) [12]. The clear difference caused by the presence of nickel is the reduction mechanism, which is a formation of reactive hydrogen on the second metal particle surface, followed by dehalogenation and hydrogenation by the reactive hydrogen [12].

$$
\begin{array}{cc}
\mathrm{Fe} \rightarrow \mathrm{Fe}^{2+}+2 \mathrm{e}^{-} & \text {(Eq. 5) } \\
2 \mathrm{H}^{+}+2 \mathrm{e}^{-} \rightarrow 2 \mathrm{H}^{*} & \text { (Eq. 6) } \\
\mathrm{RX}+2 \mathrm{H}^{*} \rightarrow \mathrm{RH}+\mathrm{HX} & \text { (Eq. 7) }
\end{array}
$$

A significant difference in reaction kinetics was also obtained among para-halogenated phenols, depending on the substituted halogens. The reaction was faster in the following order: 4-iodophenol $>$ 4-bromophenol $>4$ chlorophenol, the order of which agrees well with the corresponding average bond enthalpies. Average bond enthalpies of C-Cl, C-Br, C-I bond are 339, 276, 240 $\mathrm{KJ} / \mathrm{mol}$, respectively. This close relationship explains the reason for different reaction kinetics due to halogen atoms.

In summary, nitrate and nitrobenzene can be used to 
determine the general reduction reactivity of nZVI, while catalytic reactivity of the second metal can be determined by para-halogenated phenols. The graduated reducing reactivity towards different reducible compounds is capable of being an indicator for determining and estimating difficulties of the reaction.
This graduated reducing reactivity also agrees well with previous related literature on the potential of ZVI for different organic contaminants [3]. Depending on the desired characteristics to quantify, either removal capacity in 5 to $10 \mathrm{~min}$ or overall kinetics can be measured.

\subsection{Applicability of the method for investigation of reducing power of $\mathrm{nZVI}$ samples and reaction conditions}

The suggested methods, the reactivity test with different compounds, followed by the colorimetric assay for reduction products using a multi-well microplate, can be widely applied in the field of nZVI study. A number of nZVI samples prepared in various conditions can be tested, and it simply presents the effectiveness of prepared nZVI. Moreover, the effect of reaction conditions on nZVI reactivity can easily be simulated and determined using the suggested methods. Herein, several examples of applying the suggested method are introduced and discussed.

\subsubsection{Determining a reactivity of unknown nZVI samples}

Several commercial nZVI particles are already available on the market and these are readily used in many remediation studies. The manufacturer may provide a guideline for their usage (e.g. recommended dosage or expected reactivity); however, it may change with respect to different batches or different storage conditions. Therefore, it is sometimes hard to estimate suitable testing conditions. Prior to applying nZVI into a

As illustrated in Figure 3, commercial nZVI has a negligible reactivity towards all compounds tested. In order to accelerate reaction kinetics and promote catalytic reactivity, commercial $\mathrm{Ni} / \mathrm{Fe}$ particles were applied towards all compounds in different concentrations, 2 and $10 \mathrm{~g}-\mathrm{Fe} / \mathrm{L}$. Commercial $\mathrm{Ni} / \mathrm{Fe}$ particles provide a limited reactivity towards nitrobenzene and nitrate; product concentration was further increased by a higher commercial $\mathrm{Ni} / \mathrm{Fe}$ particles dosage. However, none of the cases exhibited production of phenol, which indicates that the role of nickel is the acceleration of nZVI oxidation by creating galvanic cells, rather than production of reactive hydrogen, in this case. remediation site, verifying the reactivity is essential to estimate appropriate remediation action.

In this study, a commercial nZVI was applied, as received, together with bimetallic $\mathrm{Ni} / \mathrm{Fe}$ particles prepared using commercial nZVI, in order to accelerate the reduction rate and also to promote catalytic reactivity. Being that the commercial nZVI was delivered several months prior to analysis, current properties should be determined.

Overall, commercial nZVI itself has reactivity towards nitrobenzene and nitrate, but the reactivity is too low to compare with lab-synthesized nZVI. It might be due to oxidation during storage or different surface characteristics caused by different synthesis procedures. It was reported that aging of nZVI was significantly dependent on its preparation method [23] and aging conditions [24]. Therefore, in order to apply the commercial nZVI tested in this study on remediation work, the use of extreme conditions, e.g. much higher iron dosage or acidic conditions, etc. should be considered.

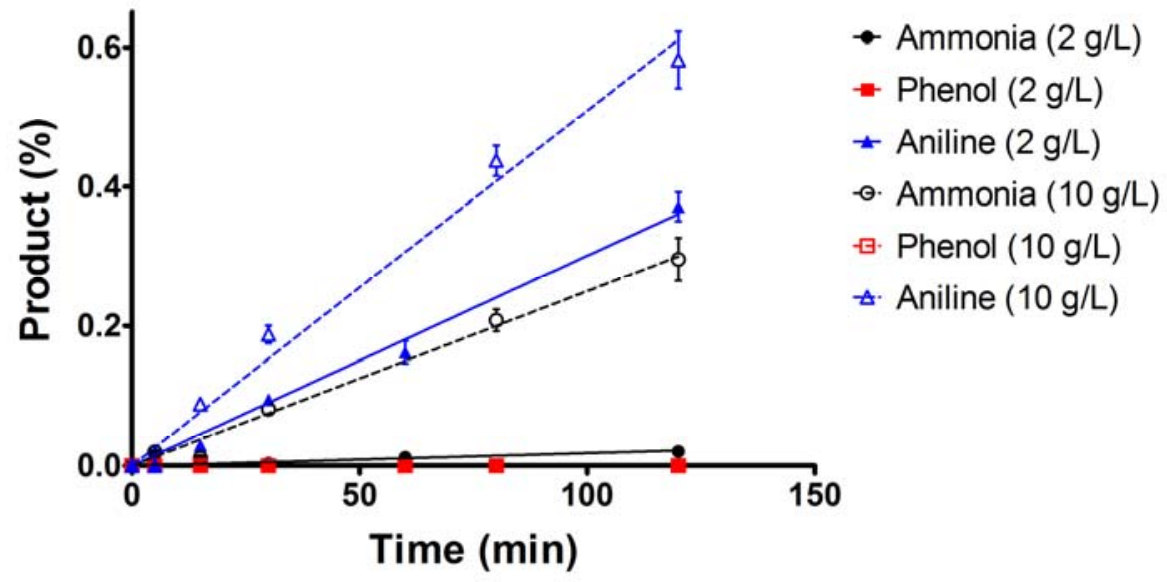

Figure 3 Generation of reaction products by reaction with commercial Ni/Fe. Note that curves are fitted with pseudo first-order kinetics. 
Table 3 Kinetic constants and half-life time derived by curve fitting via pseudo first-order kinetics (commercial nZVIs).

\begin{tabular}{|c|c|c|c|c|}
\hline$n Z V I$ & Compounds & Kinetic constant $\left(h^{-1}\right)$ & $t_{1 / 2}(\min )$ & $R^{2}$ \\
\hline Commercial Ni/Fe(2 g/L) & Nitrobenzene & 0.00181 & 23000 & 0.99 \\
\hline \multirow{2}{*}{ Commercial Ni/Fe(10 g/L) } & Nitrobenzene & 0.00319 & 13000 & 0.99 \\
\hline & Nitrate & 0.00131 & 31700 & 0.99 \\
\hline
\end{tabular}

3.3.2 Effect of bimetal contents on dehalogenation of 4-chlorophenol

Bimetallic particles have been widely applied in order to enhance nZVI reactivity, due to its catalytic behavior and higher standard redox potential promoting iron corrosion. Various transition metals having higher standard redox potentials, e.g. $\mathrm{Pd}, \mathrm{Pt}, \mathrm{Ag}, \mathrm{Ni}, \mathrm{Cu}$, etc. have been investigated. However, apart from reactivity enhancement, other aspects should also be considered (e.g. cost of the second metal, acceptable concentration of second metal in receiving water, etc.). Therefore, the effect of bimetallic contents on remediation is often discussed, and optimization is required in many cases.

Herein, the suggested method was applied to investigate the effect of bimetallic contents for dehalogenation of 4chlorophenol. The nickel content was varied over the range of $0.01-5 \%$. Figure 4 (a) illustrates the reduction of 4-chlorophenol, with respect to time, and rate constants

(a)

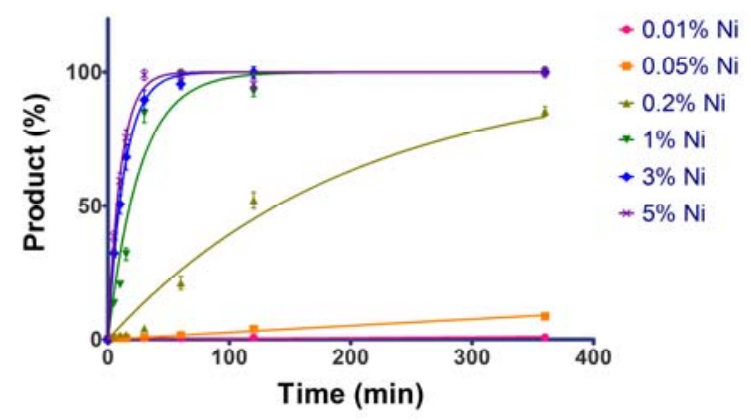

are plotted as Figure 4 (b) in log-scale. Higher Ni content generally brings about faster reaction, which clearly indicates a tremendous effect of hydrogenolysis catalyst on the dechlorination rate. However, the increase of kinetic constants is less significant above $1 \%$ of nickel content. This indicates that there is certain ratio between iron and nickel to maximize dehalogenation. When nickel mass loading is too high, the contact area between nickel and iron will be decreased gradually because the ratio of iron becomes too low, which counteracts the reactive hydrogen production, electron transport, catalytic sites, and galvanic couple formation. Similar findings could be found in a study of $\mathrm{Cu} / \mathrm{Fe}$ bimetallic particles for dechlorination [13,25]. Throughout this study, the effect of nickel contents on dehalogenation was simply and efficiently investigated. Moreover, appropriate remediation conditions could be discussed based on preliminary results showing reaction kinetics and capacity

(b)

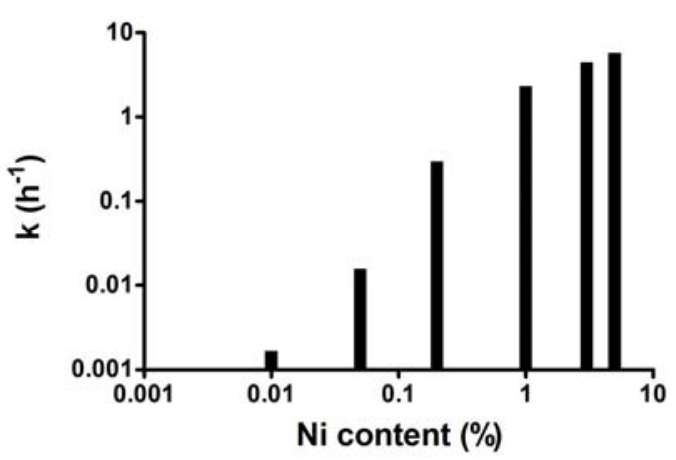

Figure 4 Effect of nickel contents on the 4-chlorophenol reduction by nZVI. (a) Generation of phenol with respect to time, and (b) derived rate constants by curve fitting in log scale. Note that curve was fitted with pseudo first-order kinetics.

3.3.3 Suggested reactivity assay procedure and its implementation

The suggested methods, those of reactivity tests combined with a colorimetric assay using a multi-well microplate, can be widely applied in the field of nZVI studies. It includes, but is not limited to, application of a stabilizer, bimetallic particles, and an electron mediator to enhance nZVI properties for environmental remediation. The overall assay can be performed in two steps: 1) batch tests with a range of substrates, and 2) analysis of reaction products by colorimetric assay, as described in Figure 5. A range of substrates is chosen based on the aim of the study. If the research focuses on investigating catalytic properties or dehalogenation activity of nZVI, a range of halophenols can be applied; whereas nitrobenzene or nitrate can be used to quantify general reduction potential of nZVI. However, it is always a good idea to test at least one of the substrates from each group (e.g. 4-chlorophenol and nitrobenzene), to confirm whether nZVI itself has the reduction capacity and the test conditions are acceptable. 
Step 1

Batch test with a range of substrate

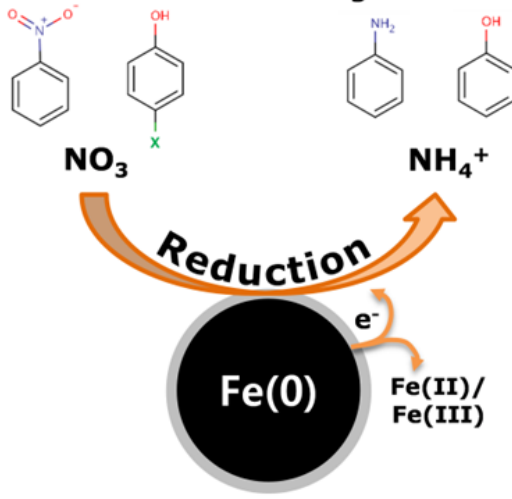

Step 2

Color assay for reaction product
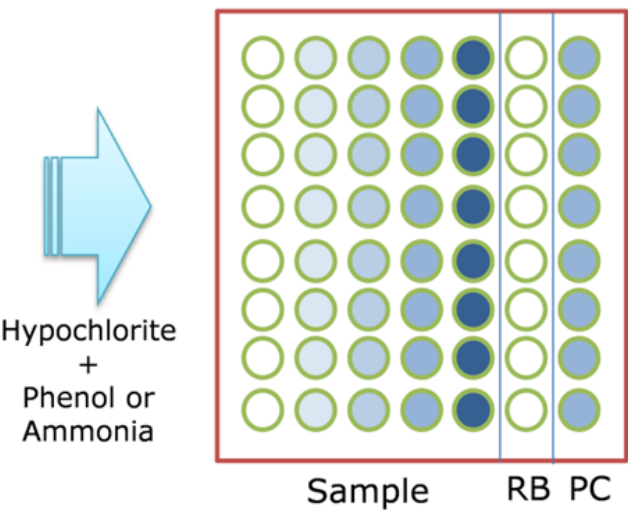

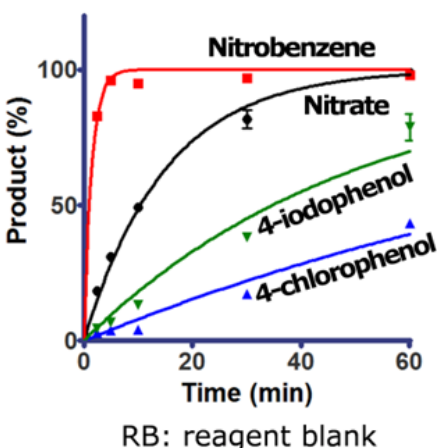

PC: positive control

Figure 5 Schematic of the suggested reactivity assay procedure

Figure 5 also explains how the colorimetric assay is designed in a 96-well microplate $(12 \times 8)$. Throughout batch tests to measure nZVI reactivity, five samples are taken, up to $1 \mathrm{~h}$ of reaction time (e.g., at 2.5, 5, 10, 30, and $60 \mathrm{~min}$ ). Analysis of samples by the color assay is always done together with a blank, reagent blank, and a positive control spiked with a known concentration of the product, to confirm the validity of the analysis. Thus, it is possible to minimize interference on the colorimetric reaction caused by constituents of the sample, e.g. buffer used or soluble metals. When the soluble iron concentration in a sample is higher than $100 \mathrm{mg} / \mathrm{L}$, sample pretreatment using sodium carbonate solution is considered, as described in previous research [17]. Therefore, the number of wells required for one analysis will be twelve, which fits with a number of wells in a row in the microplate. Several columns can be used according to the number of substrates used for the batch tests and number of replications performed. The color intensity can be quantified at the same wavelength, 680 $\mathrm{nm}$. In the standard analysis for ammonium (see section 2.1.1), the optimal wavelength for ammonia analysis was $630 \mathrm{~nm}$, but it was beneficial to use $680 \mathrm{~nm}$ as a universal quantification wavelength, since ammonia showed a much higher color intensity with respect to phenol or aniline in the concentration ranges suggested in Table 1. Therefore, the use of a microplate gives simplicity and versatility for measuring nZVI reactivity, since all analyses, including pretreatment and replication can be performed in a 96-well microplate.

\section{Conclusion}

The modified indophenol assay for determining the reductive reactivity of nZVI has been suggested. The color reaction for three different reaction products, ammonium, phenol, and aniline are simple and versatile, since the same types of reagents can be applied for all reactions. All color reactions were miniaturized into multi-well microplate, which provides a convenient and efficient assay platform. Reaction conditions (i.e. reaction time and reagent concentrations) were further optimized. A small quantity, $230 \mu \mathrm{L}$, is enough to quantify reaction products within $2 \mathrm{~h}$ of reaction time.

The three groups of compounds, nitrate, nitrobenzene, and p-halophenols, produced a graduated reactivity order, and it was possible to distinguish the reaction mechanism between normal reduction and that of the catalytic behavior of the second metal. Reaction kinetics were fast enough to complete the reaction within $1 \mathrm{~h}$. Depending on the desired characteristics to quantify, either removal capacity in 5 to $10 \mathrm{~min}$ or overall kinetics, can be selectively measured. The applicability was successfully proven by determining reactivity of an unknown nZVI sample and investigating the effect of $\mathrm{Ni}$ content with nZVI on dehalogenation. The preliminary results of nZVI reactivity obtained by the suggested assay indicate it is useful to determine suitable reaction conditions for remediation work and to estimate nZVI performance and required time. Therefore, the suggested reactivity test with different compounds, combined with a multi-well microplate based color assay, will be a useful and simple tool in various nZVI related research topics.

\section{Acknowledgement}

Yuhoon Hwang acknowledges funding for this study through individual postdoctoral grants through the Danish Council for Independent Research (DFF-4005-00393). Paul D. Mines and Henrik R. Andersen recognize funding from the Technical University of Denmark (DTU) for this work through a strategic research initiative for cooperation with KAIST. 


\section{References}

[1] F. Fu, D.D. Dionysiou, H. Liu, The use of zero-valent iron for groundwater remediation and wastewater treatment: a review., J. Hazard. Mater. 267 (2014) 194-205.

[2] T. Tosco, M. Petrangeli Papini, C. Cruz Viggi, R. Sethi, Nanoscale zerovalent iron particles for groundwater remediation: a review, J. Clean. Prod. 77 (2014) 10-21.

[3] T. Raychoudhury, T. Scheytt, Potential of zerovalent iron nanoparticles for remediation of environmental organic contaminants in water: a review., Water Sci. Technol. 68 (2013) 1425-1439.

[4] R.A. Crane, T.B. Scott, Nanoscale zero-valent iron: future prospects for an emerging water treatment technology., J. Hazard. Mater. 211-212 (2012) 112-25.

[5] Y.-H. Hwang, D.-G. Kim, H.-S. Shin, Effects of synthesis conditions on the characteristics and reactivity of nano scale zero valent iron, Appl. Catal. B Environ. 105 (2011) 144-150.

[6] Q. Wang, S. Snyder, J. Kim, H. Choi, Aqueous Ethanol modified Nanoscale Zerovalent Iron in Bromate Reduction: Synthesis, Characterization, and Reactivity, Environ. Sci. Technol. 43 (2009) 3292-3299.

[7] W. Wang, Z. Jin, T. Li, H. Zhang, S. Gao, Preparation of spherical iron nanoclusters in ethanol-water solution for nitrate removal, Chemosphere. 65 (2006) 1396-1404.

[8] N. Sakulchaicharoen, D.M. O'Carroll, J.E. Herrera, Enhanced stability and dechlorination activity of pre-synthesis stabilized nanoscale FePd particles, J. Contam. Hydrol. 118 (2010) 117-127.

[9] H. Choi, S. Agarwal, S.R. Al-Abed, Adsorption and Simultaneous Dechlorination of PCBs on GAC/Fe/Pd: Mechanistic Aspects and Reactive Capping Barrier Concept, Environ. Sci. Technol. 43 (2009) 488-493.

[10] Y. Hwang, Y.C. Lee, P.D. Mines, Y.S. Huh, H.R. Andersen, Nanoscale zero-valent iron (nZVI) synthesis in a Mgaminoclay solution exhibits increased stability and reactivity for reductive decontamination, Appl. Catal. B Environ. 147 (2014) 748-755.

[11] Y.-C. Lee, K. Lee, Y. Hwang, H.R. Andersen, B. Kim, S.Y. Lee, et al., Aminoclay-templated nanoscale zero-valent iron (nZVI) synthesis for efficient harvesting of oleaginous microalga, Chlorella sp. KR-1, RSC Adv. 4 (2014) $4122-4127$.

[12]W. ZHANG, X. QUAN, Z. ZHANG, Catalytic reductive dechlorination of p-chlorophenol in water using Ni/Fe nanoscale particles, J. Environ. Sci. 19 (2007) 362-366.

[13]R.J. Barnes, O. Riba, M.N. Gardner, T.B. Scott, S.A. Jackman, I.P. Thompson, Optimization of nano-scale nickel/iron particles for the reduction of high concentration chlorinated aliphatic hydrocarbon solutions., Chemosphere. 79 (2010) 448-54.

[14]A. Ghauch, A. Tuqan, Catalytic degradation of chlorothalonil in water using bimetallic iron-based systems., Chemosphere. 73 (2008) 751-759.

[15] S. Bae, W. Lee, Influence of riboflavin on nanoscale zero-valent iron reactivity during the degradation of carbon tetrachloride., Environ. Sci. Technol. 48 (2014) 2368-76.

[16] Y. Liu, S.A. Majetich, R.D. Tilton, D.S. Sholl, G. V. Lowry, TCE Dechlorination Rates, Pathways, and Efficiency of Nanoscale Iron Particles with Different Properties, Environ. Sci. Technol. 39 (2005) 1338-1345.

[17] Y. Hwang, P.D. Mines, M.H. Jakobsen, H.R. Andersen, Simple colorimetric assay for dehalogenation reactivity of nanoscale zero-valent iron using 4-chlorophenol, Appl. Catal. B Environ. 166-167 (2015) 18-24.

[18]A. Afkhami, R. Norooz-Asl, Micelle-mediated extraction and spectrophotometric determination of ammonia in water samples utilizing indophenol dye formation, J. Braz. Chem. Soc. 19 (2008) 1546-1552.

[19] P.L. Searle, The berthelot or indophenol reaction and its use in the analytical chemistry of nitrogen: A review, Analyst. 109 (1984) 549-568.

[20] American Public Health Association, American Water Works Association, Water Environment Federation, Standard Methods for the Examination of Water and Wastewater, 2012.

[21]K.-F. Chen, S. Li, W. Zhang, Renewable hydrogen generation by bimetallic zero valent iron nanoparticles, Chem. Eng. J. 170 (2011) 562-567.

[22] S.O. Ko, D.H. Lee, Y.H. Kim, Kinetic studies of reductive dechlorination of chlorophenols with Ni/Fe bimetallic particles., Environ. Technol. 28 (2007) 583-593.

[23] H.-S. Kim, T. Kim, J.-Y. Ahn, K.-Y. Hwang, J.-Y. Park, T.-T. Lim, et al., Aging characteristics and reactivity of two types of nanoscale zero-valent iron particles (FeBH and FeH2) in nitrate reduction, Chem. Eng. J. 197 (2012) 16-23.

[24]D. Jiang, X. Hu, R. Wang, D. Yin, Oxidation of nanoscale zero-valent iron under sufficient and limited dissolved oxygen: Influences on aggregation behaviors., Chemosphere. 122 (2015) 8-13.

[25]B. Lai, Y. Zhang, Z. Chen, P. Yang, Y. Zhou, J. Wang, Removal of p-nitrophenol (PNP) in aqueous solution by the micron-scale iron-copper (Fe/Cu) bimetallic particles, Appl. Catal. B Environ. 144 (2014) 816-830. 


\section{Supplementary Information}

\section{Graduated characterization method using a multi-well microplate for reducing reactivity of nanoscale zero valent iron materials}

Yuhoon Hwang ${ }^{1, *}$, Apostolos Salatas ${ }^{1,2}$, Paul D. Mines ${ }^{1}$, Mogens H. Jakobsen $^{3}$, Henrik R. Andersen ${ }^{1}$

\footnotetext{
${ }^{1}$ Department of Environmental Engineering, Technical University of Denmark, Miljøvej, B113, DK-2800 Kgs. Lyngby, Denmark

${ }^{2}$ Department of Environment, University of the Aegean, Xenia Building, University Hill, 81100 Mytilene, Greece

${ }^{3}$ Department of Micro- and Nanotechnology, Technical University of Denmark, Ørsteds Plads, B 345E, DK-2800 Kgs. Lyngby, Denmark
} 


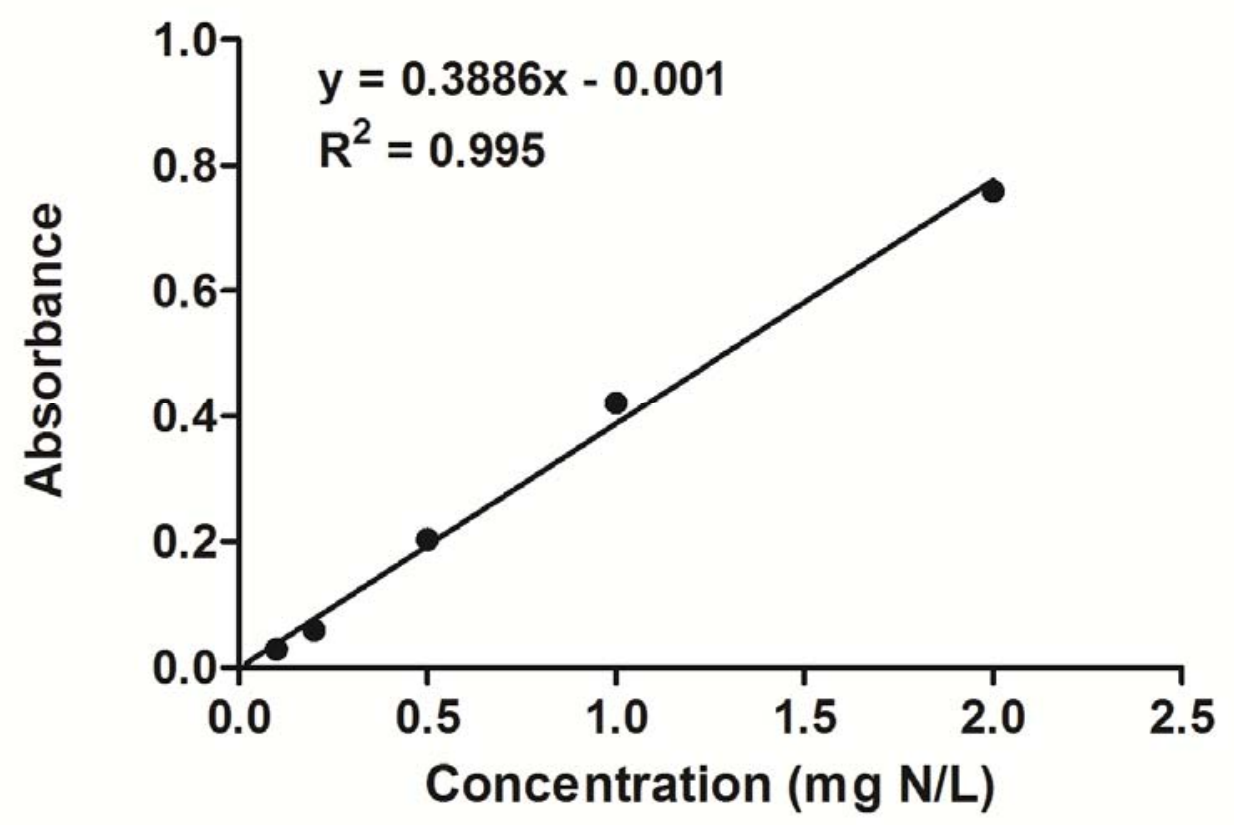

Figure S1 Standard curve for ammonium (0-2 mg-N/L) after indophenol reaction (Reaction time: $2 \mathrm{~h}$ ).

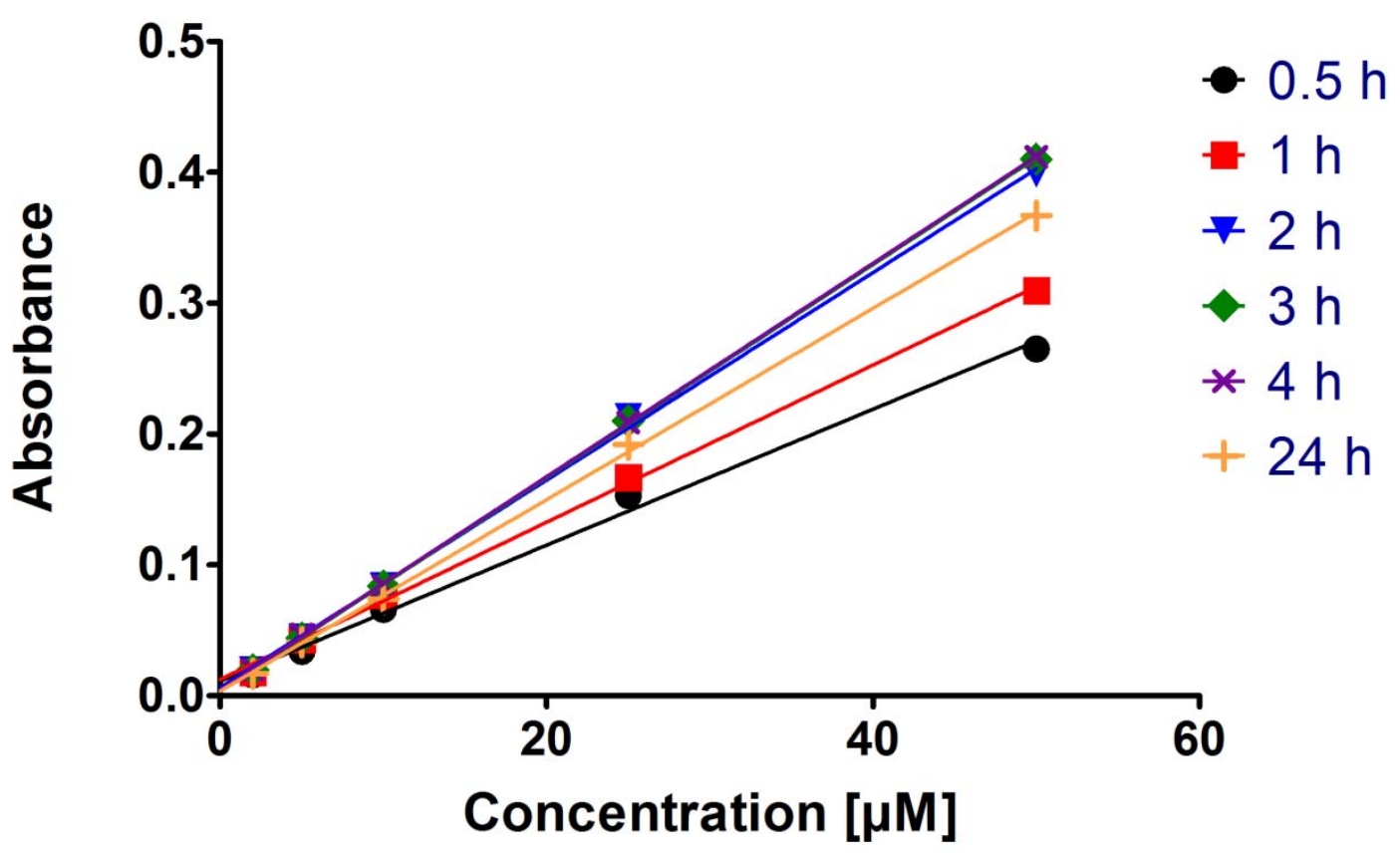

Figure S2 Standard curve for phenol $(0-50 \mu \mathrm{M})$ after reaction with $\mathrm{NH}_{2} \mathrm{Cl}$ (reagent $\mathrm{AC}$ ) in different reaction time. 


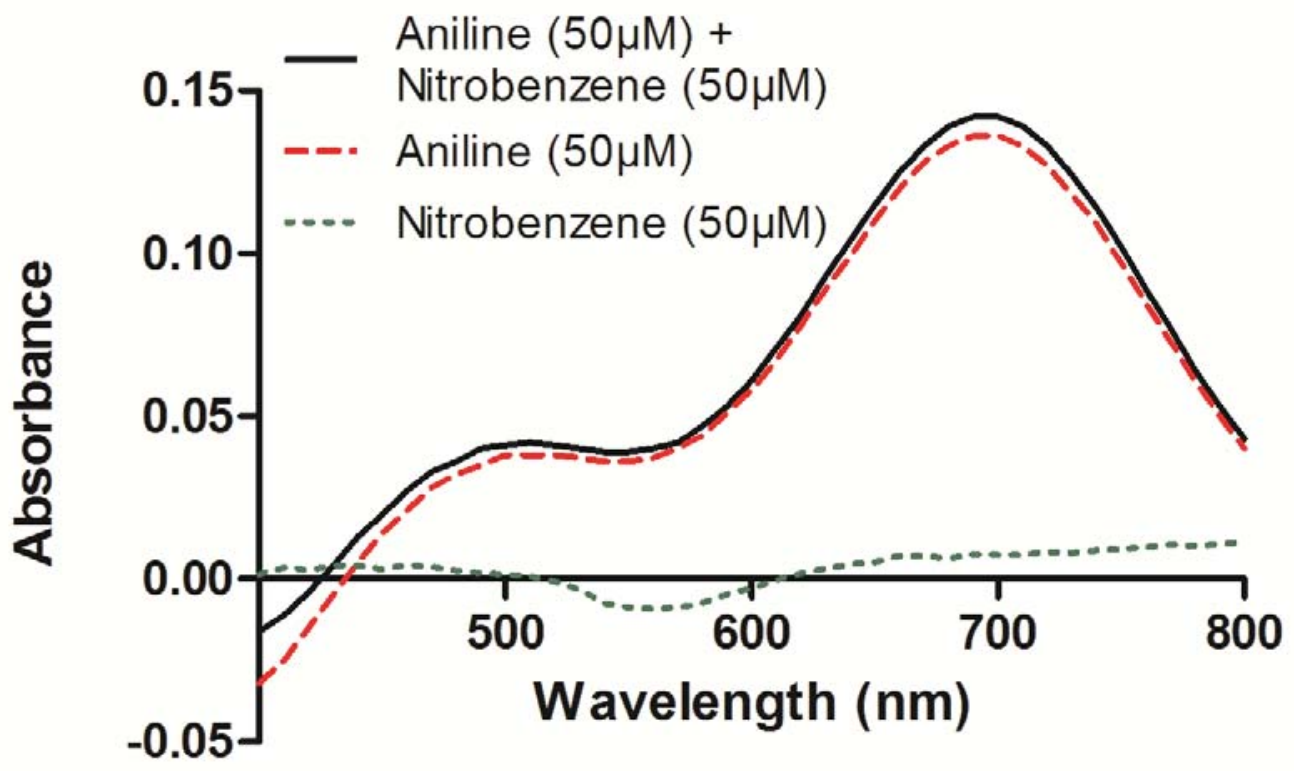

Figure S3 Spectra evolution after color reaction with reagent A and C

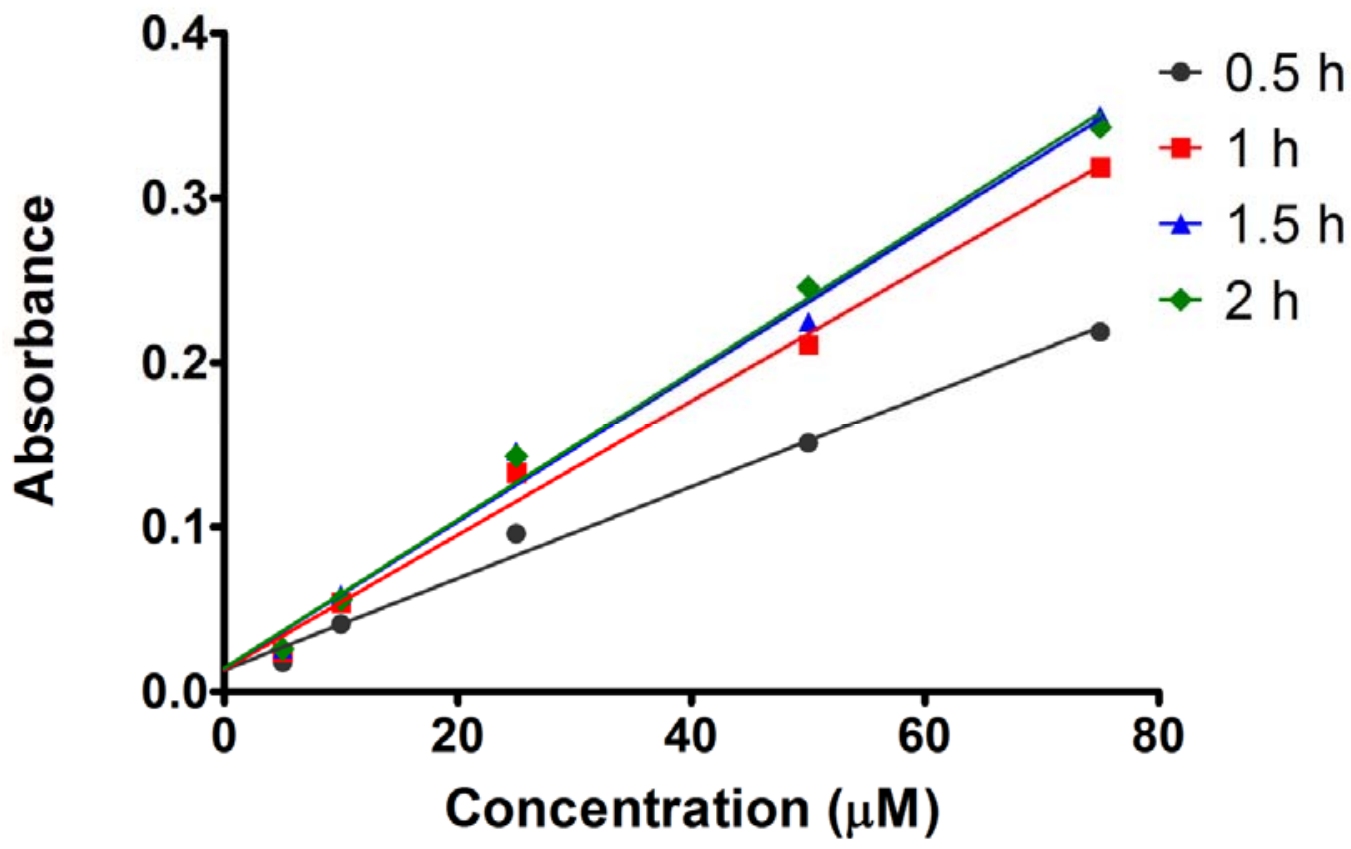

Figure S4 Standard curve for aniline $(0-75 \mu \mathrm{M})$ after reaction with reagent $\mathrm{A}$ and $\mathrm{C}$ in different reaction time. 
Table S1 Equation, LOD, and LOQ of phenol standard curves in different reaction time

\begin{tabular}{c|cccc}
\hline Time $(\boldsymbol{h})$ & Standard curve & $\boldsymbol{R}^{2}$ & LOD $(\boldsymbol{\mu M})$ & LOQ $(\boldsymbol{\mu M})$ \\
\hline $\mathbf{0 . 5}$ & $\mathrm{y}=0.0052 \mathrm{x}+0.0112$ & 1.00 & 0.5 & 1.7 \\
$\mathbf{1}$ & $\mathrm{y}=0.006 \mathrm{x}+0.0124$ & 1.00 & 0.4 & 1.4 \\
$\mathbf{2}$ & $\mathrm{y}=0.0079 \mathrm{x}+0.0063$ & 1.00 & 0.3 & 1.1 \\
$\mathbf{3}$ & $\mathrm{y}=0.0081 \mathrm{x}+0.0037$ & 1.00 & 0.3 & 1.1 \\
$\mathbf{4}$ & $\mathrm{y}=0.0082 \mathrm{x}+0.0043$ & 1.00 & 0.3 & 1.1 \\
$\mathbf{2 4}$ & $\mathrm{y}=0.0073 \mathrm{x}+0.0037$ & 1.00 & 0.4 & 1.2 \\
\hline
\end{tabular}

Table S2 Equation, LOD, and LOQ of aniline standard curves in different reaction time

\begin{tabular}{c|cccc}
\hline Time $(\boldsymbol{h})$ & Standard curve & $\boldsymbol{R}^{2}$ & LOD $(\boldsymbol{\mu M})$ & LOQ $(\boldsymbol{\mu M})$ \\
\hline $\mathbf{0 . 5}$ & $\mathrm{y}=0.0028 \mathrm{x}+0.0131$ & 0.99 & 1.6 & 5.4 \\
$\mathbf{1}$ & $\mathrm{y}=0.0041 \mathrm{x}+0.0134$ & 0.99 & 0.9 & 3.1 \\
$\mathbf{1 . 5}$ & $\mathrm{y}=0.0045 \mathrm{x}+0.0143$ & 0.99 & 1.3 & 4.3 \\
$\mathbf{2}$ & $\mathrm{y}=0.0045 \mathrm{x}+0.0123$ & 0.99 & 1.0 & 3.3 \\
\hline
\end{tabular}

